\title{
Transient Aspects of Wave Propagation Connected with Spatial Coherence
}

\author{
Ezzat G. Bakhoum ${ }^{1}$ and Cristian Toma ${ }^{2}$ \\ ${ }^{1}$ Department of Electrical and Computer Engineering, University of West Florida, 11000 University Parkway, Pensacola, FL 32514, USA \\ ${ }^{2}$ Faculty of Applied Sciences, Politechnica University, 315 Spl. Independentei, Bucharest, Romania
}

Correspondence should be addressed to Cristian Toma; cgtoma@physics.pub.ro

Received 30 June 2013; Accepted 10 July 2013

Academic Editor: Carlo Cattani

Copyright (C) 2013 E. G. Bakhoum and C. Toma. This is an open access article distributed under the Creative Commons Attribution License, which permits unrestricted use, distribution, and reproduction in any medium, provided the original work is properly cited.

\begin{abstract}
This study presents transient aspects of light wave propagation connected with spatial coherence. It is shown that reflection and refraction phenomena involve spatial patterns which are created within a certain transient time interval. After this transient time interval, these patterns act like a memory, determining the wave vector for subsequent sets of reflected/refracted waves. The validity of this model is based on intuitive aspects regarding phase conservation of energy for waves reflected/refracted by multiple centers in a certain material medium.
\end{abstract}

\section{Introduction}

As it is known, the study of light wave propagation phenomena as reflection and refraction at the interface between two different media is based on wavefronts generated by multiple centers of reflection/refraction situated on this interface. These wavefronts correspond to surfaces over which the light wave has a constant phase. Usually, a wavefront is represented by the surface over which the wave has a maximum value (the crest of a wave).

The direction of propagation for the wave (which is also the direction of the wave vector, usually denoted by $\mathbf{k}$ ) is always perpendicular to the surface of the wavefront at each point. Thus, the wavefronts of a point source (emitting in all spatial directions) are spheres, and the wave propagates radially outward the radius of a sphere being perpendicular to the circumference at each point.

According to Huygens' principle for propagation of light, each point on a certain wavefront acts as a point source that emits spherical wavelets. These wavelets propagate with the speed of light in the medium and generate the total wavefront at a later time as the envelope that encloses all of these wavelets. This corresponds to the tangent line that joins the front surface for each of them.

However, we must take into account the fact that each center of reflection/refraction (usually represented by an infinitely small spatial area of the interface) should be considered also as emitting spherical energy waves in all directions. For an oblique incidence of a plane wave, a certain center of reflection/refraction will be the first one which emits wavelets with the speed of light specific to that material medium. Until it interacts with the wavefront generated by another center of reflection/refraction, we should consider that the energy received from the incident wave is radiated along all spatial directions. After these first two wavelets interact, it can be considered that they create a wavefront with a greater radius of curvature along the main direction of reflection/refraction, resulting a lower-divergence beam. However, a significant amount of energy will still be radiated in all spatial directions. The same aspect can be noticed by analyzing the interaction of each newly created wavefront with previously reflected/refracted wavelets. The radius of curvature is lowered, but supplementary amounts of energy are still radiated in all spatial directions (not just along the main axis of reflection/refraction) by these new wavefronts. If the lowering of beam divergence is not a prevailing phenomenon, the reflected/refracted wave would vanish very quickly.

Moreover, the assumption regarding the constant phase shift ( $\pi$ for electric field $\mathbf{E}$, e.g.) for reflected/refracted wave in surface point is also questionable, since the interface is 
far from being perfectly smooth and perfectly conductive. A certain transient time interval for creating the electrostatic equilibrium is always required. As a consequence, local phase shifts for reflected/refracted wave cannot be avoided within this local mathematical model. According to the standard propagation theory of waves, these could generate multiple local waves propagating in all spatial directions. Thus, the energy of reflected/refracted wave would be dissipated in a large solid angle (corresponding to a high divergence of the light beam) and directionality would be lost within a very short length interval.

For this reason, a complete analysis of phenomena on such transient time intervals should be based on a global analysis of interface and wavefront aspects. Spatial coherence should be taken into consideration.

As a consequence, a certain amount of energy is lost during this transient time interval. A deeper analysis of this model (for the stationary regime) requires a certain memory of previous interaction and a certain spatial coherence to be taken into account, as will be shown in the next paragraphs.

\section{Periodical Effect of Momentum Space Patterns}

As it is known, quantum theory uses either position or momentum space for representing states and evolution of particles and their associated fields. A preliminary analysis of reflection/refraction phenomena is based on classical electromagnetic field, which corresponds in fact to the wave function associated to a photon (the electric field $\mathbf{E}$, magnetic field $\mathbf{B}$, vector potential $\mathbf{A}$, and scalar potential $\mathbf{V}$ being the main quantities used). This wave function can explain basic aspects in wave theory of light as reflection/refraction working within position space.

However (as it has been shown in the introduction), this theory working within position representation is suitable just for idealized cases (such as a perfectly smooth interface). It can be argued that light consists of photons which are packets of energy that primarily interact with interface atoms. Through this interaction, the energy of the photon is absorbed by collectivised electrons of the solid crystalline lattice, and the photon ceases to exist. Usually, the electron will quickly return to a lower energy state by emitting a photon. Since the photons are reemitted, not reflected or refracted, each photon behaves more like a point sourceas if the light was originating right there. At a later time, these emitted spherical waves generate the total wavefront as the envelope that encloses all these point-source waves. The effect of interface nonuniformities could be considered as vanishing by drawing a tangent line as a global approximation through the front surface for each point-source wave. Yet there is no valid argument regarding a minimum value for the radii of curvature of this tangent line. Theoretically, it could be very small, and thus the global tangent line could consist of a lot of local curves with significant curvatures which are joined together. This way a lot of divergent light beams could be created along the reflected/refracted trajectory, and the energy would disappear very quickly.
A better argument regarding the perfectly smooth approximation for reflection/refraction theory could consist in the fact that photons usually interact with collectivised electrons of the solid crystalline lattice before being reemitted. These electrons could be considered as moving tangents to the interface since sudden changes of trajectory could generate significant electromagnetic field (accelerations being involved). This picture is supported also by quantum physics, since the associated wave function for the collectivised electrons is represented in position for large space intervals, the influence of local nonuniformities being decreased. Thus, a tangent line local radii of curvature greater than a certain value can be drawn, and a better directionality for reflected/refracted wave can be obtained.

However, this explanation does not take into account the phase shift between the incident and the reemitted wave for different points of the interface. A complete analysis based on quantum theory should consider that waves reemitted from different points of the interface are part of the wavetrain corresponding to a certain photon; the probability of detecting a reflected/refracted photon is determined by the coherent plane-wave compounding method (it is well known that a particle interferes just with itself). There is no valid argument for the assumption regarding the constant phase shift between the local incident wave and the corresponding local reemitted wave in each interface point. The wave function for collectivised electrons of the crystal lattice is far from being constant in space-time along this interface. For this reason, space correlations for the incident wave-train and the reemitted wave-train should be taken into account.

It is useful to mention that a certain kind of spatial coherence can be noticed within classical electromagnetic theory, since the reflection on a perfectly conductive (metallic) interface requires the electric field $\mathbf{E}$ to vanish on this surface. For this, a certain transient time interval necessary for creating the electrostatic equilibrium is required.

As a consequence, local phase shifts for reflected/ refracted wave cannot be avoided within any local mathematical model. According to the standard propagation theory of waves, these could generate multiple local waves propagating along spatial directions which differ from the main reflection/refraction axis. Thus, the energy of reflected/refracted wave would be dissipated in a large solid angle (corresponding to a high divergence of the light beam) and directionality would be lost within a very short length interval. Thus the use of spatial coherence (based on nonlocal aspects) is justified. A harder task is to add some transient time considerations into a model based mainly on spatial correlations.

Analyzing the hypothesis of constant wave shift for the reflected/refracted wave in any point of the interface, we can observe that incident waves (parts of the associated wave corresponding to a certain photon-according to quantum mechanics) for first time interact with spatially extended functions/patterns defined on the interface material medium (crystal lattice with specific quantum functions for collectivised electrons and for phonons-quanta corresponding to spatially extended vibrations). This could suggest that lattice quantum functions interact in a global manner with parts of the incident wave on a large spatial area of the interface, 
a certain correlation between phase of reflected/refracted wave being noticed even for surface points separated by possible crystal defects. This implies that a kind of support wave is generated on this interface, which acts upon reflecting/refracting points and correlates the phase of reemitted waves by interface points situated at great distance.

This aspect regarding space correlations achieved within a very short time interval for nonadjacent spatial intervals which interact with wavefronts (part of the reflected/refracted wave) becomes a key issue if we consider that reflected/refracted wave can undergo diffraction at a later time. The requirement of constant phase shift for parts of the associated wave generated by points or edges of a diffraction grating is still valid-yet the points or edges of such a diffraction grating are nonadjacent and cannot be correlated by any surface quantum wave functions. So, the support waves previously mentioned should propagate with high speed in space in order to regroup the wavefronts into a light beams with certain directionality, so as the diffracted wave doesnot vanish within a short time interval after interaction with the diffraction grating.

For connecting nonadjacent spatial areas, the position space representation for associated wave function is no more recommended. However, the associated wave corresponds to the same photon (defined on a certain wavelength interval and on a certain three-dimensional wave vector interval). This implies that the use of momentum space representation is suitable for interconnecting these nonadjacent space intervals where a certain material medium interacts with parts of the same incident or reflected/refracted wave-train (an example being the case of a later diffraction when a limited number of high-intensity directions are created). With a certain periodicity, this momentum representation becomes active and generates momentum (wave vector) values. For nonadjacent space intervals, the phase correlation within this momentum representation should be achieved through highspeed propagating support waves. The momentum values generated when this representation is active represents the base for wave propagation on subsequent time intervals, when the position representation becomes active.

We must check whether such high speed support waves can be considered using the standard wave equation. As it is known, the homogeneous wave equation in free space (in a space-time point without any sources) in one dimension (when movement is restricted along the $O x$-axis) is represented by

$$
\frac{\partial^{2} \phi}{\partial x^{2}}=\frac{1}{v^{2}} \frac{\partial^{2} \phi}{\partial t^{2}},
$$

where $v$ corresponds to the wave velocity. The standard mathematical solution is represented by

$$
\phi(x, t)=\phi_{f}(x-v t)+\phi_{r}(x+v t),
$$

where $\phi_{f}(x-v t)$ corresponds to the forward wave (which moves towards $x=+\infty$ as time $t$ increases) and $\phi_{r}(x+v t)$ corresponds to the reverse wave (which moves towards $x=-\infty$ as time $t$ increases).
The three-dimensional homogeneous wave equation in free space (in a space-time point without any sources) is represented by

$$
\frac{\partial^{2} \phi}{\partial x^{2}}+\frac{\partial^{2} \phi}{\partial y^{2}}+\frac{\partial^{2} \phi}{\partial z^{2}}=\frac{1}{v^{2}} \frac{\partial^{2} \phi}{\partial t^{2}} .
$$

For spherical coordinates, considering that the wave function $\phi$ is invariant under rotations (the case when spherical waves are emitted from a certain point in space or are convergent towards a central point), the wave equation for $\phi$ (depending only on distance $r$ to this central point and time) can be written as

$$
\frac{\partial^{2} \phi}{\partial r^{2}}+\frac{2}{r} \frac{\partial \phi}{\partial r}=\frac{1}{v^{2}} \frac{\partial^{2} \phi}{\partial t^{2}} .
$$

The standard mathematical solutions for this case are

$$
\phi(r, t)=\frac{1}{r} \phi_{d}(r-v t)+\frac{1}{r} \phi_{c}(r+v t),
$$

where $\phi_{d}(r-v t)$ corresponds to a divergent wave (which moves from a certain point outwards along all radial directions as time $t$ increases) and $\phi_{c}(x+v t)$ corresponds to a convergent wave (which moves from all radial directions towards a certain point as time $t$ increases).

However, these standard solutions are not suitable for our purpose. Their velocity is limited by the $v$ parameter of the wave equation. Yet we can notice that the threedimensional homogeneous wave equation admits solutions under the following form:

$$
\phi(x, y, z, t)=a x+b y+c x+d t+e,
$$

where quantities $a, b, c, d, e$ are constant values. This corresponds to a time-dependent plane equation - a plane which moves in the three-dimensional space. Let us suppose that $\phi$ equals $e$ in the origin, at the zero moment of time (this means $x=y=z=t=0$ ). At this zero moment of time, this value corresponds also (according to standard analytical geometry) to a spatial plane defined by the following:

$$
a x+b y+c x+0 d+e=e,
$$

which means that

$$
a x+b y+c x=0
$$

(the spatial origin being included in this plane). At a later time $t$, the same value $e$ could be noticed for $\phi$ in a plane defined by the following:

$$
e=a x+b y+c x+d t+e,
$$

which means

$$
a x+b y+c x+d t=0 .
$$

At this time moment $t$, the distance from spatial origin to this plane (parallel to the plane defined at zero moment, since 
quantities $a, b, c$ corresponding to a vector normal to the plane do not vary in time) equals

$$
D=\left|\frac{0 a+0 b+0 c+(d t)}{\sqrt{a^{2}+b^{2}+c^{2}}}\right|=\left|\frac{d t}{\sqrt{a^{2}+b^{2}+c^{2}}}\right| .
$$

This can be also written as

$$
\frac{D}{t}=\left|\frac{d}{\sqrt{a^{2}+b^{2}+c^{2}}}\right|,
$$

which means that the plane on which $\phi$ equals $e$ passing through origin at zero time moment which moves with velocity

$$
w=\frac{D}{t}=\left|\frac{d}{\sqrt{a^{2}+b^{2}+c^{2}}}\right|
$$

in space. The propagation velocity depends only on $a, b, c, d$ constants. Since there is no restriction regarding the choice for these constant values, it results that (unlike standard solutions of wave equation) these polynomials can be represented as constant values propagating in space-time with any velocity (this physical quantity being no more correlated to the wave equation). Since the velocity has no more an upper limit, these polynomials can be put in correspondence with the high-speed support wave requested for performing practically an instant correlation between different nonadjoining space intervals interacting with the same associated wave (the values in momentum space representation being the same).

\section{Conclusions}

This study has presented transient aspects of light wave propagation connected with spatial coherence. It has been shown that reflection and refraction phenomena involve spatial patterns which are created within a certain transient time interval. After this transient time interval, these patterns (connected to spatial coherence) act like a certain memory, determining the wave vector for subsequent sets of reflected/refracted waves. The validity of this model is based on the study of wavefronts generated by multiple centers of reflection/refraction situated on the interface-the total wavefront at a later time being the envelope that encloses all of these wavelets. Analyzing the hypothesis of constant wave shift for the reflected/refracted wave in any point of the interface, it is shown that this should be connected with a nonlocal mathematical model which takes into account

(i) that incident waves (parts of the associated wave corresponding to a certain photon-according to Quantum Mechanics) the first time interaction with spatially extended functions/patterns defined on the interface material medium (crystal lattice with specific quantum functions for collectivised electrons and for phonons-quanta corresponding to spatially extended vibrations);

(ii) that these kinds of spatially extended functions are generated also for nonadjacent spatial intervals which interact with wavefronts (part of the reflected/refracted wave) at a later time.
The first aspect is not surprising (since a spatially extended wave function for the interface can be easily be defined using quantum considerations). However, the second is a novel aspect which implies the use of momentum space representation for defining the same values for parts of the same reflected/refracted associated wave-train in nonadjacent space intervals (an example being the case of a later diffraction).

This extended spatial model can explain why minor different local shifts do not appear, since (according to standard wave propagation theory) these could lead to multiple local waves propagating along spatial directions which differ to the main reflection/refraction axis. Thus, the entire reflected/refracted wave would be dissipated in a large solid angle (corresponding to a high divergence of the light beam) and directionality would be lost within a very short length interval.

The mathematical aspects are based on first order polynomials satisfying the wave equation. Unlike standard solutions of wave equation, these polynomials can be represented as constant values propagating in space-time with any velocity (this physical quantity being no more correlated to the wave equation). Since the velocity has no more upper limit, these polynomials can be put in correspondence with certain support waves able to perform practically an instant phase correlation between different non-adjoining space intervals interacting with the same associated wave. This correlation should be achieved within momentum space representation for the associated wave, since within this representation all values corresponding to the same wave-train are the same in nonadjacent space intervals.

These linear functions differ from time dynamics based on temporal coherence (as presented in [1]). Being constant values propagating in space time, they can be put in correspondence with traveling wavelets inside a certain material medium as in $[2,3]$. The difference consists in the fact that these functions correspond to linear space-time functions which are active with a certain periodicity. Phase aspects of quantum interactions were also presented in [4], without any spatial correlations to be mentioned. A nonlinear model for creating spatial patterns (using nonlinear equations) has been presented in [5]. Scale spatial aspects were also presented in [6], in opposition to temporal noise aspects generating uncorrelation presented in $[7,8]$ by the same group of researchers.

\section{References}

[1] E. G. Bakhoum and C. Toma, "Dynamical aspects of macroscopic and quantum transitions due to coherence function and time series events," Mathematical Problems in Engineering, vol. 2010, Article ID 428903, 13 pages, 2010.

[2] J. J. Rushchitsky, C. Cattani, and E. V. Terletskaya, "Wavelet analysis of the evolution of a solitary wave in a composite material," International Applied Mechanics, vol. 40, no. 3, pp. 311-318, 2004.

[3] C. Cattani, "Multiscale analysis of wave propagation in composite materials," Mathematical Modelling and Analysis, vol. 8, no. 4, pp. 267-282, 2003. 
[4] E. G. Bakhoum and C. Toma, "Mathematical transform of traveling-wave equations and phase aspects of quantum interaction," Mathematical Problems in Engineering, vol. 2012, Article ID 695208, 15 pages, 2010.

[5] F. Doboga, "Different structural patterns created by short range variations of internal parameters," in Computational ScienceICCS 2007, vol. 4488 of Lecture Notes in Computer Science, pp. 1060-1066, 2007.

[6] M. Li, Y. Q. Chen, J. Y. Li, and W. Zhao, "Holder scales of sea level," Mathematical Problems in Engineering, vol. 2012, Article ID 863707, 22 pages, 2012.

[7] M. Li and W. Zhao, "On bandlimitedness and lag-limitedness of fractional Gaussian noise," Physica A, vol. 392, no. 9, pp. 19551961, 2013.

[8] M. Li and W. Zhao, "On 1/f noise," Mathematical Problems in Engineering, vol. 2012, Article ID 673648, 23 pages, 2012. 


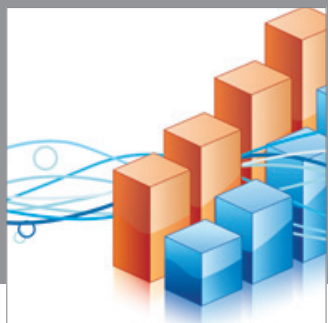

Advances in

Operations Research

mansans

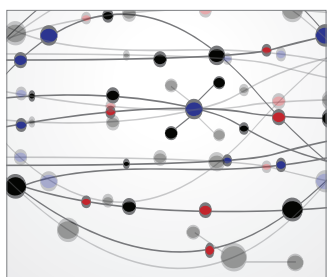

The Scientific World Journal
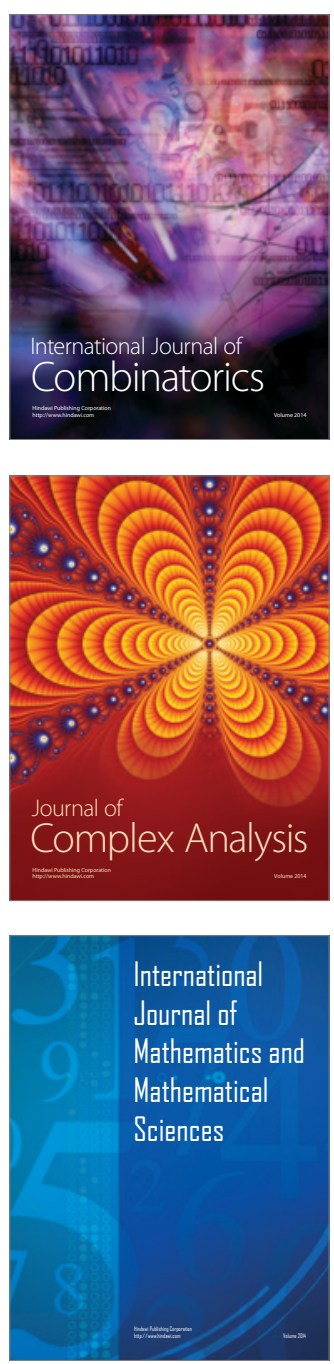
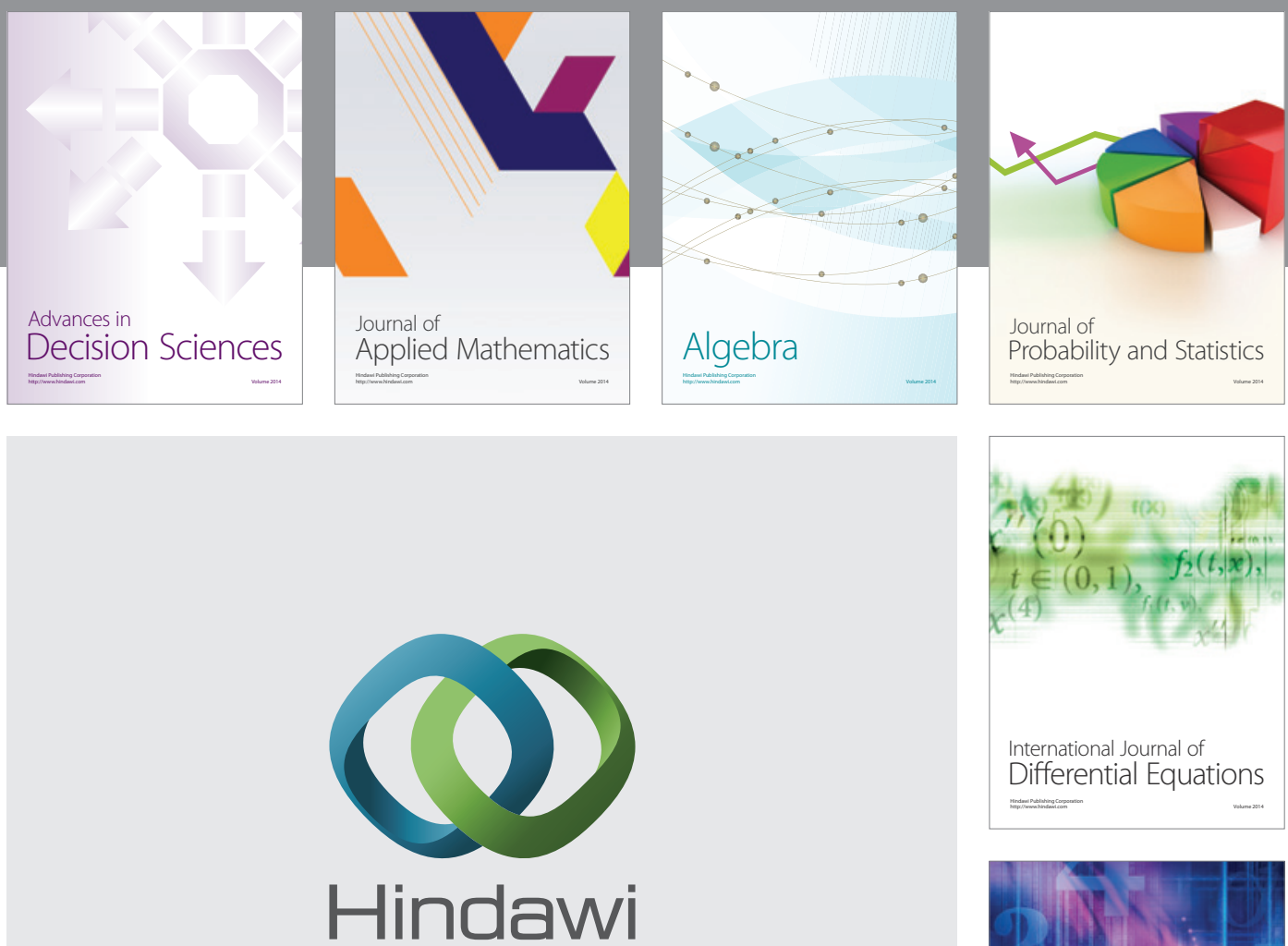

Submit your manuscripts at http://www.hindawi.com
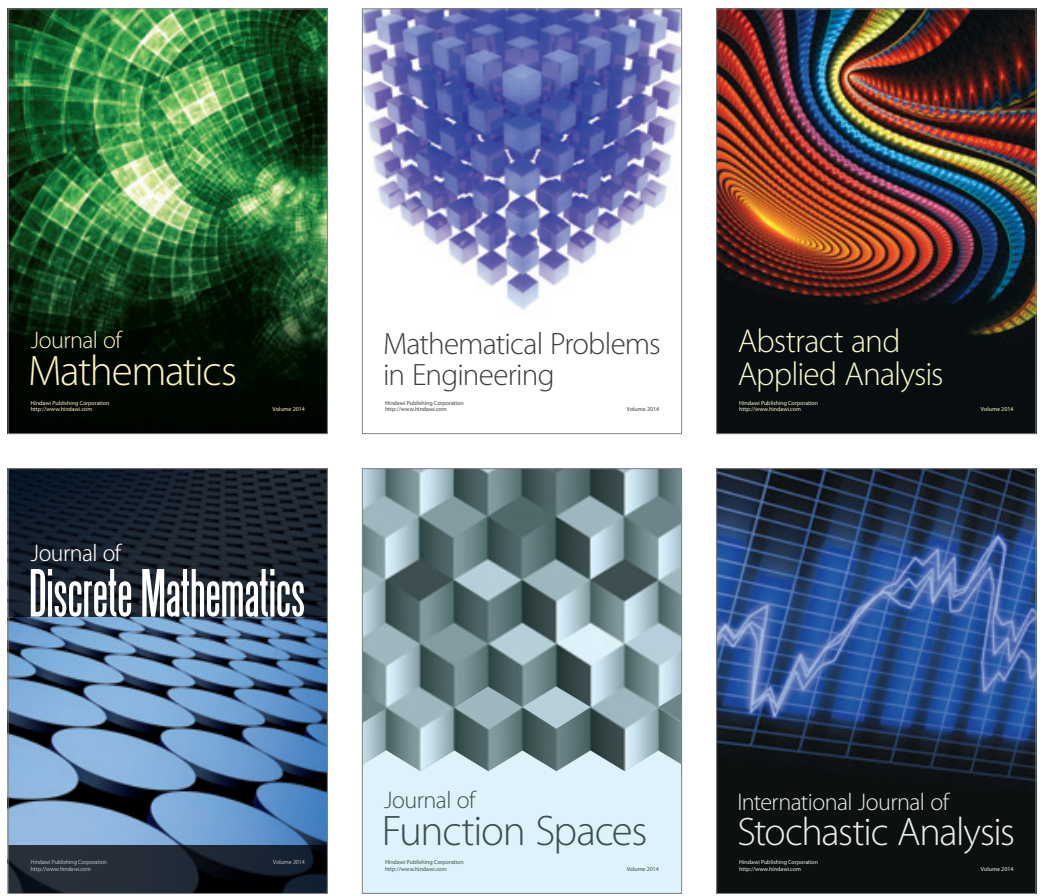

Journal of

Function Spaces

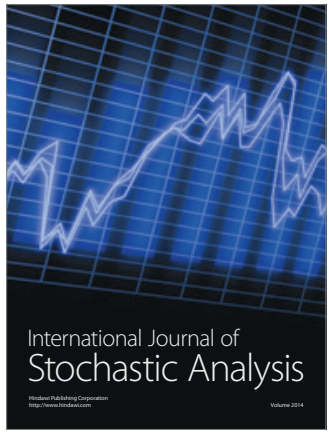

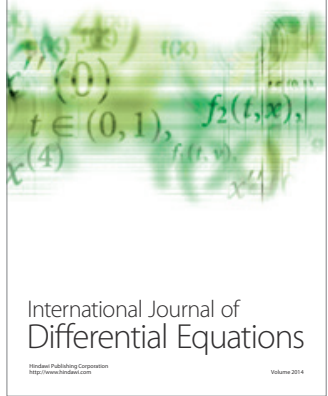
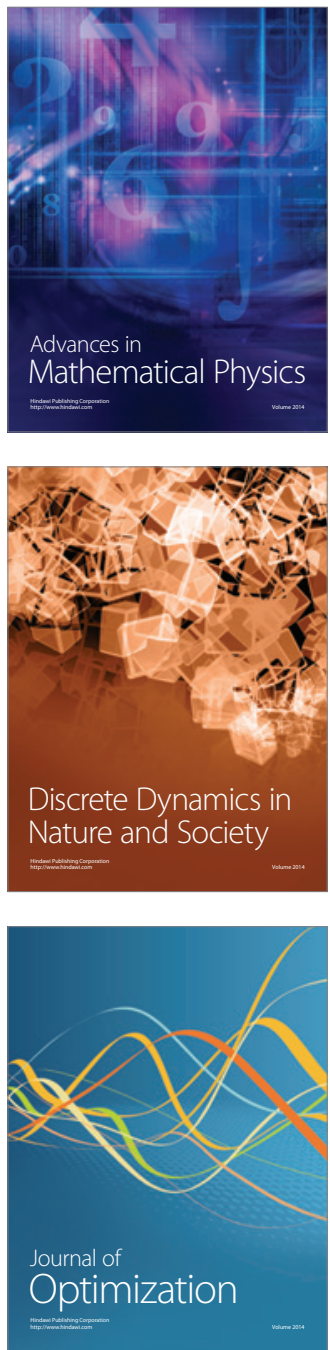